\title{
Amphionides reynaudii (H. Milne Edwards, 1832) (Amphionidacea: Amphionididae): identificación de estadios larvales y nuevo orden de crustáceos en aguas oceánicas chilenas
}

\author{
Jorge Rivera ${ }^{1}$, Guillermo Guzmán ${ }^{1}$ \& Armando Mujica ${ }^{2}$ \\ ${ }^{1}$ Departamento de Ciencias del Mar, Universidad Arturo Prat, Casilla 121, Iquique, Chile \\ ${ }^{2}$ Facultad de Ciencias del Mar, Universidad Católica del Norte, Casilla 117, Coquimbo, Chile \\ e-mail: rivera_mon@hotmail.com
}

\begin{abstract}
RESUMEN. Se identifican algunos estadios larvales de Amphionides reynaudii, único representante del orden Amphionidacea el cual se registra por primera vez para aguas oceánicas chilenas. Los ejemplares fueron colectados durante el desarrollo de los cruceros Cimar efectuados a las islas oceánicas chilenas, en una transecta entre Caldera e isla de Pascua (primavera de 1999) y alrededor del archipiélago de Juan Fernández e islas Desventuradas (primavera de 2000). Se discute la presencia de este orden de crustáceos en aguas del océano Pacífico sudoriental y se incluye una clave de identificación para su reconocimiento.
\end{abstract}

Palabras claves: Amphionides reynaudii, larvas, distribución, Pacifico sudoriental.

\section{Amphionides reynaudii (H. Milne Edwards, 1832) (Amphionidacea: Amphionididae): identifications of larval stages and new order of crustaceans of Chilean oceanic waters}

\begin{abstract}
Some larval stages of the Amphionides reynaudii are identified, only representative of the Order Amphionidacea are registed for the first time for Chilean oceanic waters. The specimens were collected during the development of the cruises to the Chilean oceanic islands, in a transect between Caldera and Easter Island (spring 1999) and Juan Fernandez archipelago and Desventuradas Islands (spring 2000). The presence of this order of crustaceans in the waters of the southeastern Pacific ocean is discussed and an identification key for their recognition in the plankton samples is included.
\end{abstract}

Key words: Amphionides reynaudii, larvae, distribution, southeastern Pacific.

El actual orden de crustáceos eucáridos Amphionidacea, fue propuesto sobre la base de la descripción de las formas larvales por H. Milne Edwards (1832). Sin embargo, permanece en dudas su status actual debido a la escasa representación de estadios adultos y su condición de especie pelágica cosmopolita, sobre la cual se ha descrito en la literatura un par de especies en dos géneros, Amphionides y Amphion. No obstante, algunos autores señalan que solo existiría una especie, quedando el género originalmente descrito por H. Milne Edwards, el cual correspondería a un estado juvenil avanzado (H. Milne Edwards, 1832; Gurney, 1936; Bowman \& Abele, 1982; MacLaughlin, 1982; Schram, 1986).

Actualmente, Amphionidacea se reconoce como un orden aparte de los decápodos (Bowman \& Abele, 1982; Martin \& Davis, 2001), grupo en el cual fueron originalmente asignados, debido a su aparente relación con los sergéstidos o bien con alguna forma postlarval de un erionaceo del género Polycheles sensu Boas (1879). Como se mencionó anteriormente, la aparentemente única especie es de distribu- 
ción cosmopolita, pero su presencia ha sido registrada en escasas localidades geográficas. El objetivo principal del presente trabajo es entregar información sobre la identificación de algunos estadios larvales de Amphionides reynaudii y registrar su presencia para la fauna carcinológica chilena dentro del Pacífico sudoriental.

El material objeto de estudio se obtuvo en dos cruceros oceanográficos efectuados a las islas oceánicas chilenas, el crucero Cimar 5 efectuado en una transecta entre Caldera $\left(27^{\circ} 00^{\prime} \mathrm{S}, 70^{\circ} 52^{\prime} \mathrm{W}\right)$ e isla de Pascua $\left(27^{\circ} 10^{\prime} \mathrm{S}, 109^{\circ} 20^{\prime} \mathrm{W}\right)$ en primavera de 1999 y el crucero Cimar 6 efectuado alrededor del archipiélago de Juan Fernández (334ㄴ's, $\left.80^{\circ} 51^{\prime} \mathrm{W}\right)$ y de las islas Desventuradas $\left(26^{\circ} 16^{\prime} \mathrm{S}\right.$, $80^{\circ} 04^{\prime} \mathrm{W}$ ) en primavera de 2000 . En ambos cruceros se tomaron muestras de zooplancton con redes Bongo de $59 \mathrm{~cm}$ de diámetro y $300 \mu \mathrm{m}$ de abertura de malla, y con una red IKMT (Isaac-Kid Midwater Trawl) de $9 \mathrm{~m}^{2}$ de abertura de boca, túnel de $10 \mathrm{~m}$ de largo con malla $10 \mathrm{~mm}$ y copo de $1000 \mu \mathrm{m}$, las cuales se arrastraron entre los 0-200 y los 0-700 a $1000 \mathrm{~m}$ de profundidad respectivamente. En las muestras se colectó una serie de crustáceos y componentes zooplanctónicos de aguas superficiales y mesopelágicas, entre los que se encontraron 13 ejemplares de $A$. reynaudii.

\section{Amphionides reynaudii (Fig. 1)}

Material examinado: 13 ejemplares capturados durante los cruceros Cimar 5 y Cimar 6 (Tabla 1), correspondientes a diferentes estadios de desarrollo larval, los cuales están depositado en la colección de referencia del Museo del Mar de la Universidad Arturo Prat de Iquique. Los estadios larvales coinciden con las características morfológicas específicas descritas por Heegaard (1969) para las larvas de esta especie, aunque difieren en las tallas observadas con respecto a las registradas por este autor para cada estadio (Tabla 2).

Diagnosis de la larva Amphion: caparazón con rostro pequeño y proceso dorsal anterior, espinas orbitales y antenales. Pleurobranquias y hepatopáncreas aparecen en los estadios más desarrollados. Ojos con pedúnculo corto y globos oculares grandes, ovalados y facetados. Antenas con escafocerito y flagelo antenular desarrollado. Anténulas tan largas como el escafocerito antenal, en los primeros estadios unirramas con el segmento basal casi cinco veces más largo que el segmento distal el cual soporta estetos apicales; el flagelo antenular se for- ma en los últimos estadios de desarrollo. Apéndices bucales, mandíbula, maxílula, maxila y primer maxilípedo (toracópodo1) ampliamente separados del resto de los apéndices toráxicos y están bien desarrollados en los estadios larvales, reduciéndose completamente los dos primeros en los individuos adultos. Toracópodos 2 y 3 (maxilípedos 2 y 3 ) desarrollados, con largos exópodos setosos y funcionales desde los primeros estadios. El resto de los toracópodos se forma gradual y correlativamente durante los diferentes estadios de desarrollo, terminando con siete pares en las hembras y ocho en los machos. Abdomen con seis segmentos, primer segmento con un par de procesos laterales pequeños en los últimos estadios. Telson amplio, margen posterior redondeado con un par de setas plumosas más pequeñas que el resto en la línea media durante los primeros estadios, aguzándose y formando un margen posterior trunco con dos espinas pequeñas, perdiendo las setas plumosas durante los últimos estadios. Urópodos bajo la cutícula del telson en los dos primeros estadios y posteriormente libres, llegando a ser tan largos como el telson en los últimos estadios de desarrollo.

Distribución: durante el crucero Cimar 5 se capturaron 10 larvas de Amphionides reynaudii desde aproximadamente $\operatorname{los} 85^{\circ} \mathrm{W}$ hasta $\operatorname{los} 95^{\circ} \mathrm{W}$ a lo largo del trayecto entre Caldera e isla de Pascua. Posteriormente, durante el crucero Cimar 6 se registraron solo dos larvas, una en la isla Alejandro Selkirk (archipiélago de Juan Fernández) y la otra en la isla San Félix (islas Desventuradas).

Según lo señalado en la literatura, esta especie es netamente pelágica y de amplia distribución, habitando en casi todos los océanos, particularmente en aguas tropicales y subtropicales entre $\operatorname{los} 35^{\circ} \mathrm{N} \mathrm{y}$ $35^{\circ} \mathrm{S}$ (Holthuis, 1993). El primer registro de las larvas de $A$. reynaudii en el Pacífico sudoriental corresponde al área de los montes submarinos de la dorsal de Nazca e isla Salas y Gómez (Vereschaka, 1995), lo cual coincide con el presente registro pero amplía su distribución oriental para aguas del archipiélago de Juan Fernández e islas Desventuradas.

Heegaard (1969) señala que la distribución de las larvas de $A$. reynaudii tendrían un patrón diferente entre los océanos Atlántico, Índico y Pacífico, siendo éstas mucho más numerosas en el Atlántico que en los otros dos océanos. Los registros actuales para el Pacífico sudoriental también reflejan una tendencia similar, siendo escasas las larvas captura- 

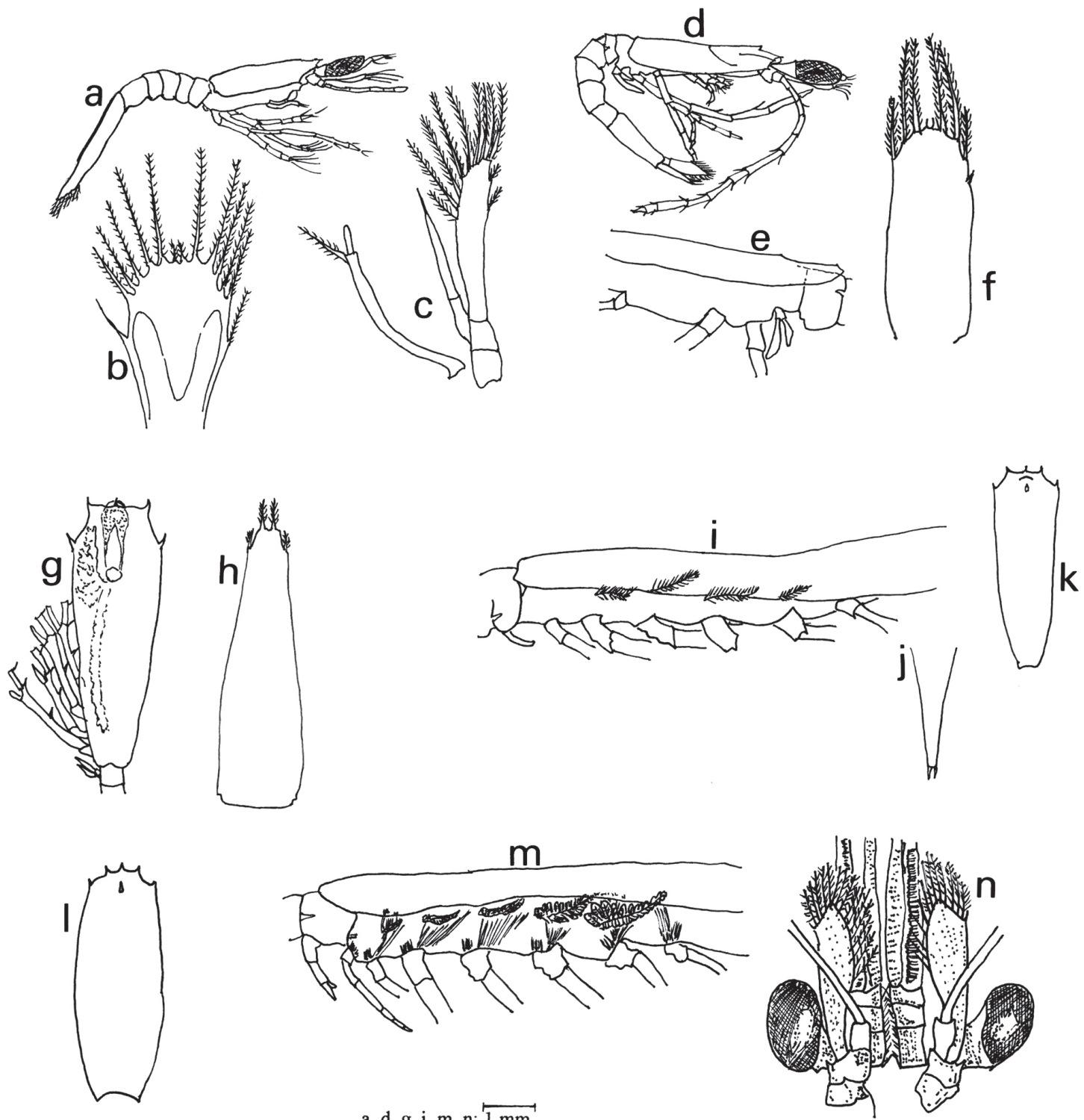

$$
\begin{aligned}
& \text { a, d, g, i, m, n: } 1 \mathrm{~mm} \\
& \text { b, f, h: } 0,5 \mathrm{~mm} \\
& \text { c: } 0,1 \mathrm{~mm} \\
& \text { k, } 1: 2 \mathrm{~mm}
\end{aligned}
$$

Figura 1. Esquemas de larvas y apéndices de Amphionides reynaudii. a-c) Amphion 2; d-f) Amphion 4; g-h) Amphion 8; i-k) Amphion 12 (hembra); l-n) Amphion 13 (macho). a) vista lateral, b) telson, c) antena y anténula, d) vista lateral, e) vista lateral del tórax, f) telson, g) vista dorsal del caparazón, h) telson, i) vista lateral del tórax, j) telson, $k$ ) vista dorsal del caparazón, l) vista dorsal del caparazón, $m$ ) vista lateral de los toracópodos, n) vista antero-ventral del tórax (redibujados de Heegaard, 1969).

Figure 1. General scheme of larvae and appendages of Amphionides reynaudii. a-c) Amphion 2; d-f) Amphion 4; g-h) Amphion 8; i-j) Amphion 12 (female); k-m) Amphion 13 (male). a) lateral view, b) telson, c) antenna y antennule, d) lateral view, e) lateral view of the thorax, f) telson, g) dorsal view of the carapace, h) telson, i) lateral view of the thorax, $\mathrm{j}$ ) telson; $k$ ) dorsal view of the carapace, l) dorsal view of the carapace $m$ ) lateral view of the thoracopods, n) antero-ventral view of the thorax (redraw after Heegaard, 1969). 
Tabla 1. Ubicación de las estaciones donde se colectaron larvas de Amphionides reynaudii.

Table 1. Location of stations where the Amphionides reynaudii larvae were collected.

\begin{tabular}{|lcllllll|}
\hline Crucero & Estación & Localidad & Latitud (S) & Longitud (W) & Fecha & $\begin{array}{c}\text { Número de ejemplares } \\
\text { Bongo }\end{array}$ \\
IKMT
\end{tabular}

Tabla 2. Comparación del grado de desarrollo y longitud total (LT) de las larvas de Amphyonides reynaudii colectadas en el presente estudio con los registros de Heegaard (1969). N: número de larvas, ( ) toracópodos rudimentarios, * longitud cefalotórax.

Table 2. Comparison of the development degree and the total longitude (LT) of the larval specimens of Amphyonides reynaudii in this study with the Heegaard (1969) reports. N: larvae number, ( ) rudiment thoracopods, * carapace length.

\begin{tabular}{|ccc|ccc|c|}
\hline \multicolumn{3}{|c|}{ Presente estudio } & \multicolumn{3}{|c|}{ Heegaard (1969) } & Williamson (1973) \\
\hline $\mathbf{N}$ & Toracópodos & LT (mm) & Estadio & Toracópodos & LT (mm) & Estadios \\
\hline & - & - & Mysis I & 3 & 4,0 & Zoea 1 \\
& - & - & Mysis II & 3 & 5,5 & Zoea 2 \\
2 & $3(4)$ & $6,12-6,24$ & Mysis III & $3(4)$ & 5,8 & Zoea 3 \\
& - & & Mysis IV & $3(4)$ & 6,0 & Zoea 4 \\
2 & $3(4)$ & $8,00-8,10$ & Mysis V & $4(5)$ & 6,5 & Zoea 5 \\
1 & 4 & 10,20 & Mysis VI & $4(5)$ & 7,0 & Zoea 7 \\
1 & $4(5)$ & 13,08 & Mysis VII & $5(6)$ & 8,0 & Zoea 8 \\
1 & 5 & 14,50 & Mysis VIII & $5(6)$ & 9,0 & Zoea 9 \\
- & - & - & Mysis IX & $6(7)$ & 9,5 & Hembra \\
1 & $6(7)$ & 18,48 & Mysis X & $6(7)$ & 13,0 & Hexual \\
3 & 7 & $19,50-20,15$ & Mysis XI & 7 & 17,0 & Macho \\
1 & $7(8)$ & 22,16 & Mysis XII & 7 & 23,0 & 25,0 \\
1 & 8 & $20,20 *$ & Mysis XIII & 8 & \\
\hline
\end{tabular}

das tanto por Vereschaka (1995) como las del presente trabajo. Sin embargo, esto puede ser producto de la escasa investigación realizada en aguas oceánicas del Pacífico suroriental.

Los registros de adultos de esta especie han sido muy escasos en aguas superficiales, ya que habitan preferentemente en aguas profundas alrededor de los 2.000 m de profundidad o más (Holthuis, 1993). Sin embargo, las larvas se han colectado entre los 30$500 \mathrm{~m}$, existiendo una migración ontogenética de los estadios más avanzados hacia aguas profundas (Gurney, 1936; Heegaard, 1969; Williamson, 1973).

Sistemática y estado actual del conocimiento: la posición actual del orden Amphionidacea dentro de los crustáceos eucáridos (Bowman \& Abele, 1982; Martin \& Davis, 2001), ha estado en discusión des- de los primeros registros y descripciones de la única especie perteneciente a este orden, debido principalmente a sus formas larvales, las cuales inicialmente fueron atribuidas a larvas de decápodos carideos (Gurney, 1936; Heegaard, 1969).

Las larvas de $A$. reynaudii son parecidas a las zoeas de los decápodos carideos; pero, las postlarvas, juveniles y adultos son completamente diferentes de este grupo, siendo ésta la base tomada por Williamson (1973) para el actual reconocimiento de Amphionidacea como un orden aparte de los decápodos y eufáusidos, pero dentro de los eucáridos por tener larvas comparables morfológicamente. No obstante, este autor reconoce que los estadios larvales de $A$. reynaudii podrían ser producto de una hibridación y transferencia larval desde los carideos (Williamson \& Rice, 1996). 
Por otra parte, Schram (1986) también separa a Amphionodacea sobre la base de caracteres morfológicos, como la carencia del epipodito en el segmento basal del primer maxílipedo, excluyéndolos de los pleociemados a pesar de tener filobranquias sobre las pleuras, siendo estas características típicas de este último grupo de decápodos.

La mayor parte de la investigación realizada sobre este grupo ha estado remitida a la morfología y desarrollo larval, destacando el trabajo de Gurney (1936) que describe algunos estadios tempranos y avanzados de larvas Amphion, estimando un desarrollo larval de nueve estadios. Posteriormente, Heegaard (1969) realiza una descripción detallada y completa, determinando 13 estadios larvales y 2 postlarvales, caracterizando además ejemplares adultos de esta especie.

Sin embargo, no hay un total acuerdo en cuanto al número de estadios larvales de la secuencia completa registrada por Heegaard (1969), donde Williamson (1973) señala que los estadios 12 y 13 de Heegaard serían la hembra y el macho respectivamente, señalando un claro dimorfismo sexual a partir del estadio 9. Éstos se diferenciarían por la presencia de un octavo par de toracópodos unirramos y más pequeños en el macho, y por la carencia de este par en las hembras, con solo siete pares y con el primer par de pleópodos unirramos y modificados, armados marginalmente con setas, los cuales se extienden por debajo del tórax para formar una cámara incubatriz (Williamson, 1973).

Los toracópodos de las larvas de Amphion, constituyen un carácter que puede generar confusión, debido a la disposición que presentan dentro del cefalotórax, caracterizándose principalmente, por la amplia separación entre el primer y segundo par de toracópodos, tanto en los estadios larvales como adultos (Fig. 1). Los tres primeros pares de toracópodos se denominan comúnmente maxilípedos (McLaughlin, 1982). No obstante, las diagnosis y descripciones morfológicas para los adultos de Amphionides reynaudii, caracterizan a esta especie y a su respectivo orden por tener un solo par de maxilípedos (toracópodo 1), siendo los toracópodos 2 y 3 funcionales para locomoción y no para alimentación (McLaughlin, 1982; Holthuis, 1993).

Las larvas analizadas en este trabajo también reflejan un desarrollo gradual. Esto se observa en la aparición de los toracópodos 4-8 (pereiópodos), aunque éstos difieren en cuanto a las longitudes totales medias registradas por Heegaard (1969) para cada estadio (Tabla 2). Esta situación confirma en cierta medida lo señalado por Williamson (1973), en que los estadios larvales de $A$. reynaudii pueden variar morfológicamente en diferentes regiones y en diferentes individuos en una misma región. Estas variaciones también se han detectado en otros grupos, como eufáusidos y en algunos decápodos con desarrollo extendido (Williamson, 1982).

\section{Clave general para la identificación de larvas de Amphionides reynaudii en aguas oceánicas del Pacífico sudoriental.}

1 - Forma general de camarón, branquias no cubiertas por el cefalotórax............Euphausiacea

- Forma general de camarón, branquias cubiertas por el cefalotórax ........................................ 2

2 - Toracópodos 1 y 2 ampliamente separados ..3

- Toracópodos 1 y 2 no separados ..................... 4

3 - Pleonito 2 no sobrepuesto al pleonito 1; telson espatulado con borde posterior ovalado en los estadios tempranos y aguzado en los estadios avanzados, con dos pequeñas espinas terminales; sin toracópodos quelados o subquelados en los estadio larvales

.Amphionidacea (larva amphion)

- Pleonito 2 sobrepuesto o no sobrepuesto al pleonito 1.............................. Decapoda.... 4

4 - Pleonito 2 no sobrepuesto al pleonito 1; telson fuertemente hendido en los estadios tempranos y con más de dos espinas en los estadios avanzados; toracópodos 4-6 quelados en los últimos estadios. Dendrobranchiata

- Pleonito 2 siempre sobrepuesto en el pleonito 1; telson bilobulado o triangular en los estadios tempranos y con los bordes laterales paralelos, con más de dos espinas o setas en los estadios avanzados; toracópodos 4-5 quelados o subquelados en los últimos estadios de desarrollo Caridea

\section{AGRADECIMIENTOS}

Los autores expresan sus sinceros agradecimientos al Comité Oceanográfico Nacional de Chile por el financiamiento y desarrollo de los cruceros de investigación científica marina Cimar 5 y 6 , que han permitido la elaboración de trabajos que contribuyen al conocimiento de la biodiversidad marina. 


\section{REFERENCIAS}

Boas, J. 1879. Amphion und Polycheles (Willemoesia). Zool. Anz., 11: 256-259.

Bowman, T. \& L. Abele. 1982. Classification of the recent Crustacea. En: D.E. Bliss (ed.). The Biology of Crustacea. Academic Press, New York, 1: 1-25.

Gurney, R. 1936. Larvae of decapod Crustacea. Part III. Phyllosoma. Discovery Rep., 12: 400-440.

Heegaard, P. 1969. The larvae of decapod Crustacea. The Amphionidae. Dana Rep., 77: 1-82.

Holthuis, L. 1993. The recent genera of the Caridean and Stenopodidean shrimp (Crustacea, Decapoda), with an appendix on the order Amphinodacea. Leiden National Naturhistorisch Museum, 328 pp.

McLaughlin, P. 1982. Comparative morphology of recent Crustacea. W.H. Freeman \& Company, San Francisco, 177 pp.

Recibido: 21 octubre 2003; Aceptado: 2 abril 2004
Martin, J. \& G. Davis. 2001. An updated classification of the recent Crustacea. Nat. Hist. Mus. Los Angeles County, Sci. Ser., 39: 132 pp.

Milne Edwards, H. 1832. Note sur un nouveau genre de Crustacés de l'ordre des Stomatopodes. Ann. Soc. Ent, France, 1: 336-340.

Schram, F. 1986. Crustacea. Oxford University Press, London, 606 pp.

Vereschaka, A. 1995. Macroplankton in the nearbottom layer of continental slopes and seamounts. Deep Sea Res., 42(9): 1639-1668.

Williamson, D. 1973. Amphionides reynaudii $(\mathrm{H}$. Milne Edwards), representative of a proposed new order of eucaridan Malacostraca. Crustaceana, 25: 35-50.

Williamson, D. 1982. Larval morphology and diversity. En: D.E. Bliss (ed.). The Biology of Crustacea. Academic Press, New York, 2: 43-110.

Williamson, D. \& A. Rice. 1996. Larval evolution in the Crustacea. Crustaceana, 69(3): 267-287. 